\title{
Trials Evaluating Ticagrelor in Cardiovascular Disease: Will It Reign Supreme in the Anti-Platelet World? \\ Cheuk Kit Wong*
}

Department of Medicine and Therapeutics, Chinese University of Hong Kong, Sha Tin, Hong Kong

${ }^{*}$ Corresponding author: Wong CK, Department of Medicine and Therapeutics, Faculty of Medicine, Prince of Wales Hospital Shatin, Chinese University of Hong Kong, Sha Tin, Hong Kong, Tel: 852-26323139; E-mail: cheuk-kit.wong@cuhk.edu.hk

Received date: February 19, 2017; Accepted date: February 24, 2017; Published date: February 27, 2017

Copyright: (c) 2017 Wong CK. This is an open-access article distributed under the terms of the Creative Commons Attribution License, which permits unrestricted use, distribution, and reproduction in any medium, provided the original author and source are credited.

\section{Editorial}

Ticagrelor and prasugrel are newer and stronger platelet P2Y12 receptor antagonists than their predecessor clopidogrel. Clopidogrel is a pro-drug requiring a 2 -step hepatic activation processes. Speed of activation varies according to the cytochrome P-450 2C19 allele [1,2]. Ticagrelor is a direct non-thienopyridine P2Y12 antagonist producing equal or stronger inhibition of the P2Y12 receptor even when compared to the newer thienopyridine prasugrel [3].

\section{The PLATO Trial}

In the PLATO trial of 18624 patients with acute coronary syndrome on background aspirin therapy, ticagrelor $90 \mathrm{mg}$ twice daily significantly reduced all-cause and cardiovascular mortality as compared to clopidogrel $75 \mathrm{mg}$ daily (4.5\% vs. $5.9 \%$ and $4.0 \%$ vs. $5.1 \%$ respectively over 1-year) [4]. In PLATO, the Kaplan-Meier curves showing the adjudicated primary end point (a composite of death from vascular causes, myocardial infarction, or stroke) separated in the first month and kept diverging throughout the year. These positive findings held true in the very large subgroup of patients with coronary stent implanted, and ticagrelor reduced stent thrombosis more as compared to clopidogrel [5]. Ticagrelor treated patients had higher nonprocedural but not CABG-related or fatal bleeding compared to clopidogrel treated patient [4], perhaps an expected finding given that ticagrelor is a stronger but reversible P2Y12 antagonist (with faster offset of action) while clopidogrel is a weaker but non-reversible P2Y12 antagonist.

In PLATO a geographical regional interaction was noted (with interaction $\mathrm{P}$ value of 0.05 ) where outcome trended in opposite direction between patients randomized in North America $(n=1814)$ and patients randomized elsewhere. In these 1814 patients, outcome tended to be worse with ticagrelor than with clopidogrel (HR 1.25, 95\% CI 0.93-1.67). Exploratory analysis revealed only one factor-aspirin dose (higher in North America than elsewhere) that might explain the observed regional interaction [6]. Currently, the advantage from ticagrelor as compared with clopidogrel is believed to be associated only with the use of low-dose $(<100 \mathrm{mg})$ aspirin, and guidelines specify this point.

\section{Dual antiplatelet therapy post coronary stenting}

Previous editorials in the Journal have reviewed the basis for dual antiplatelet therapy post coronary stenting $[1,2]$. In a very recent patient level meta-analysis of 11,473 patients with coronary stenting (58.5\% with stable coronary disease) from 6 randomized trials, patients with $\leq 6$ month dual antiplatelet therapy tended to have higher 1-year event rates of myocardial infarction or stent thrombosis than patients with 1-year dual antiplatelet therapy (HR 1.48; 95\% CI, 0.98-2.22) if they were stented for unstable disease; while rates were similar if they were stented for stable disease (HR 0.93; 95\% CI, 0.65-1.35; interaction $\mathrm{P}$ value $=0.09$ ). By network meta-analysis, 3-month but not 6-month dual antiplatelet therapy was associated with higher event rates in the former patients whereas no significant differences were apparent in the latter patients. However, shorter duration dual antiplatelet therapy was associated with lower rates of major bleeding compared with 1-year dual antiplatelet therapy. Mortality was not different. These two findings held true irrespective of the clinical reason for stenting [7].

Apart from the indication for stenting, the risk for stent thrombosis depends also on the nature of the stent design with newer generation drug eluting stents having generally less thrombogenicity (thinner stent struts, fluoropolymer or newer biodegradable polymers, nonpaclitaxel eluting) than their older counterparts [2].

\section{From PLATO to DAPT}

In PLATO, all patients had acute coronary syndrome with about $1 / 3$ having ST elevation myocardial infarction. Event curves are diverging throughout the first year, raising questions as to whether they would continue to do so if the trial extended beyond one year and whether prolonged dual antiplatelet therapy is advantageous.

The DAPT trial [8] evaluated prolonged dual antiplatelet therapy after coronary stenting. Patients that had tolerated well a year of dual antiplatelet therapy with aspirin and thienopyridine ( $n=9961,2 / 3$ clopidogrel, $1 / 3$ prasugrel) were randomly assigned to continue receiving the thienopyridine or to receive placebo for another 18 months while continuing long-term aspirin therapy. In these 9961 patients, $26 \%$ presented initially with acute myocardial infarction and $51 \%$ had $>1$ clinical or lesion-related risk factor for stent thrombosis. Over the treatment period from 12 to 30 months post-stenting, continued thienopyridine (as compared with placebo) reduced the major composite endpoint of death, myocardial infarction, or stroke ( $4.3 \%$ vs. $5.9 \%$; HR, $0.71 ; 95 \%$ CI, 0.59 to 0.85 ). However, all-cause mortality was higher at $2.0 \%$ with thienopyridine versus $1.5 \%$ with placebo (HR 1.36, 95\% CI, 1.00 to 1.85). The rate of moderate or severe bleeding was also increased with thienopyridine $(2.5 \%$ vs. $1.6 \%$, $\mathrm{P}=0.001$ ). In both groups there was an elevated risk of stent thrombosis and myocardial infarction in the 3 months after stopping thienopyridine treatment.

The DAPT findings reflect the competing risks of ischemia/ thrombosis versus bleeding, despite the fact that patients had already tolerated a year of dual antiplatelet therapy prior to randomization. For ticagrelor, the relative bleeding risks could be different as reflected in PLATO comparing aspirin-ticagrelor with aspirin-clopidogrel. 


\section{From PLATO to PEGASUS}

In PLATO, the benefit from dual aspirin-ticagrelor therapy over aspirin-clopidogrel was observed in both invasively managed and noninvasively managed patients [4,9]. With unstable coronary disease there is usually a coronary culprit lesion and potentially other active non-culprit vascular segments. The PLATO findings [4,9] might also suggest protection from ticagrelor on non-culprit, non-stented coronary segments. Whether ticagrelor benefited patients with stable coronary disease was tested in PEGASUS-a 3-arm 21162 patient trial comparing long-term therapy with ticagrelor (2 randomized doses) versus placebo on top of low-dose aspirin. All patients had a history of myocardial infarction $>1$ year prior to enrolment [10].

The two ticagrelor arms (90 mg twice daily and $60 \mathrm{mg}$ twice daily) each significantly reduced, as compared with placebo, the primary composite endpoint of cardiovascular death, myocardial infarction, or stroke at 3 years ( $7.85 \%$ with $90 \mathrm{mg}, 7.77 \%$ with $60 \mathrm{mg}$, and $9.04 \%$ with placebo). Rates of TIMI major bleeding were higher with ticagrelor (2.60\% with $90 \mathrm{mg}$ and $2.30 \%$ with $60 \mathrm{mg}$ ) than with placebo (1.06\%), but intracranial hemorrhage or fatal bleeding occurred equally $(0.63 \%$, $0.71 \%$, and $0.60 \%$, respectively). Mortality was $5.15 \%$ with $90 \mathrm{mg}$ ticagrelor, $4.69 \%$ with $60 \mathrm{mg}$ ticagrelor and $5.16 \%$ with placebo. Increased cardiovascular protection from dual aspirin-ticagrelor over aspirin alone could have been balanced off by increased bleeding.

\section{From PLATO to EUCLID}

Aspirin has been recommended for cardiovascular disease for decades. Aspirin often causes gastrointestinal side effects including bleeding. Intracranial bleeding is rarer but more dreadful. The PEGASUS findings may be considered an anti-climax highlighting the increased bleeding risks and the lack of major mortality benefit for combined low-dose aspirin and ticagrelor. The question remains as to whether stand-alone ticagrelor (without aspirin) will benefit patients with stable coronary disease.

EUCLID [11] was partly based on the CAPRIE trial reported $>2$ decades ago [12] where clopidogrel $75 \mathrm{mg}$ daily slightly out-performed aspirin $325 \mathrm{mg}$ daily as anti-platelet mono-therapy in reducing cardiovascular events. The superiority of clopidogrel was more pronounced in patients with peripheral artery disease-one of the 3 subgroups tested in CAPRIE. In EUCLID, 13885 patients with symptomatic peripheral artery disease were randomized in a doubleblind manner to receive mono-therapy with ticagrelor $(90 \mathrm{mg}$ twice daily) or with clopidogrel (75 mg once daily), without background aspirin. EUCLID excluded patients with unstable coronary disease. Over 30 months, the primary efficacy end point (composite of adjudicated cardiovascular death, myocardial infarction, or ischemic stroke) occurred in $10.8 \%$ of patients with ticagrelor and $10.6 \%$ with clopidogrel $(\mathrm{P}=0.65)$. In each group, acute limb ischemia occurred in $1.7 \%$ of the patients $(\mathrm{P}=0.85)$ and major bleeding in $1.6 \%(\mathrm{P}=0.49)$.

Both EUCLID and PLATO used the same primary efficacy endpoint for the randomized comparison of clopidogrel versus ticagrelor. The negative findings from EUCLID are at odds with the positive results from PLATO. Unlike PLATO, EUCLID had an important exclusion-a poor clopidogrel metabolizer status for the cytochrome P-450 2C19 allele, defined as a genotype with two loss-of-function alleles. As explained, clopidogrel is a pro-drug and will be inert in the homozygous "poor" metabolizers. Such polymorphism is more common in certain racial groups such as Asians and Blacks [1]. In
EUCLID, 616 patients were deemed homozygous with two loss-offunction alleles leaving 13885 patients randomized.

\section{The future of ticagrelor for atherosclerotic cardiovascular disease}

As previously discussed in the Journal [13] randomized controlled trials provide the gold standard of evidence-based medicine. The plethora of findings from clinical trials discussed herein has provided ample information. Equally important is the mechanistic basis for the selection of a particular P2Y12 receptor antagonist. This is a fast evolving field.

Some years ago it was questioned whether measuring platelet reactivity after clopidogrel could be reaching the end of the road [1] given that the more efficacious $\mathrm{P} 2 \mathrm{Y} 12$ receptor antagonist prasugrel and ticagrelor were becoming available. Today, platelet P2Y12 receptors can be immediately and completely blocked by intravenous cangrelor for ad-hoc PCI procedures and this parenteral P2Y12 receptor blockade is followed by oral blocker.

Last year commenting on the ANTARCTIC trial [2], a suggestion was made that in super-responders to prasugrel (through point-of-care platelet reactivity measurement 14 days post coronary stenting) downtitrating to clopidogrel might be cost-saving. In this editorial, the EUCLID finding that clopidogrel in "good" metabolizers produced as good results as ticagrelor is highlighted.

With ticagrelor, its relatively quicker onset of action and potency (as compared to other oral P2Y12 blockers) enable urgent PCI to be performed more safely upon new angiographic findings, but both attributes are inferior to effects from intravenous cangrelor. Cangrelor has very quick offset of action. In contrast, the antiplatelet effect of tricagrelor will persist over 3 days (clopidogrel 5 days, prasugrel 7 days) after the last dose deferring CABG if that is deemed the revascularization option after angiography.

As mentioned, evaluating the bleeding risks is essential to judge the benefit-to-risk ratio of any dual antiplatelet therapy including combined aspirin-ticagrelor therapy. The side effects of ticagrelor causing dyspnea and bradyarrhythmia are unique among the P2Y12 blockers and should be watched for. What remain to be determined are whether stand-alone ticagrelor will outperform aspirin or aspirinticagrelor combination in long-term therapy for stable coronary disease, and whether it will do so for unstable disease (managed medically or with stenting) after an initial period of dual antiplatelet therapy.

\section{References}

1. Wong CK (2012) Measuring Platelet Reactivity after Clopidogrel-Has it Reached the End of the Road. Cardiol Pharmacol 1: e105.

2. Wong CK (2016) From Arctic to Antarctic: Is There any Remaining Role for Platelet Function Testing to Tailor Therapy after Coronary Stent Implantation. Cardiovasc Pharm Open Access 5: e132.

3. Angiolillo DJ, Curzen N, Gurbel P (2014) Pharmacodynamic evaluation of switching from ticagrelor to prasugrel in patients with stable coronary artery disease: results of the SWAP-2 Study (Switching Anti Platelet-2). J Am Coll Cardiol 63: 1500-1509.

4. Wallentin L, Becker RC, Budaj A (2009) PLATO Investigators. Ticagrelor versus clopidogrel in patients with acute coronary syndromes. N Engl J Med 361: 1045-1057.

5. Steg PG, Harrington RA, Emanuelsson H (2013) Stent thrombosis with ticagrelor versus clopidogrel in patients with acute coronary syndromes: an 
Citation: Wong CK (2017) Trials Evaluating Ticagrelor in Cardiovascular Disease: Will It Reign Supreme in the Anti-Platelet World? Cardiovasc Pharm Open Access 6: e133. doi:10.4172/2329-6607.1000e133

Page 3 of 3

analysis from the prospective, randomized PLATO trial. Circulation 128 1055-1065.

6. Mahaffey KW, Wojdyla DM, Carroll K (2011) Ticagrelor compared with clopidogrel by geographic region in the Platelet Inhibition and Patient Outcomes (PLATO) trial. Circulation 124: 544-554.

7. Palmerini T, Della RD, Benedetto U (2017) Three, Six, or Twelve Months of Dual Antiplatelet Therapy After DES Implantation in Patients With or Without Acute Coronary Syndromes: An Individual Patient Data Pairwise and Network Meta-Analysis of Six Randomized Trials and 11,473 Patients. Eur Heart J.

8. Mauri L, Kereiakes DJ, Yeh RW (2014) DAPT Study Investigators. Twelve or 30 months of dual antiplatelet therapy after drug-eluting stents. N Engl J Med 371: 2155-2166.

9. Lindholm D, Varenhorst C, Cannon CP (2014) Ticagrelor vs. clopidogrel in patients with non-ST-elevation acute coronary syndrome with or without revascularization: results from the PLATO trial. Eur Heart J 35: 2083-2093.
10. Bonaca MP, Bhatt DL, Cohen M (2015) PEGASUS-TIMI 54 Steering Committee and Investigators. Long-term use of ticagrelor in patients with prior myocardial infarction. N Engl J Med 372: 1791-1800.

11. Hiatt WR, Fowkes FG, Heizer G (2017) EUCLID Trial Steering Committee and Investigators. Ticagrelor versus Clopidogrel in Symptomatic Peripheral Artery Disease. N Engl J Med 376: 32-40.

12. CAPRIE Steering Committee (1996) A randomised, blinded, trial of clopidogrel versus aspirin in patients at risk of ischaemic events (CAPRIE). CAPRIE Steering Committee Lancet 348: 1329-1339.

13. Wong CK (2016) Time for a reappraisal: How much has the last 10 years of "mainstream" STEMI research impacted on STEMI outcome. Cardiovasc Pharm Open Access 4: e129. 